\title{
Complete plasmid sequence carrying type IV-like and type VII secretion systems from an atypical mycobacteria strain
}

\author{
Sergio Mascarenhas Morgado/ ${ }^{+}$, Michel Abanto Marín, Fernanda S Freitas, \\ Erica Lourenço Fonseca, Ana Carolina Paulo Vicente
}

Fundação Oswaldo Cruz-Fiocruz, Instituto Oswaldo Cruz, Laboratório de Genética Molecular de Microrganismos, Rio de Janeiro, RJ, Brasil

The genus Mycobacterium is highly diverse and ubiquitous in nature, comprehending fast- and slow-growing species with distinct impact in public health. The plasmid-mediated horizontal gene transfer represents one of the major events in bacteria evolution. Here, we report the complete sequence of a 160,489 bp circular plasmid (pCBMA213_2) from an atypical and fast-growing environmental mycobacteria. This is a unique plasmid, in comparison with the characterised mycobacteria plasmids, harboring a type IV-like and ESX-P2 type VII secretion systems. pCBMA213 2 can be further explored for evolutionary and conjugation studies as well as a tool to manipulate DNA within this bacteria genus.

Key words: fast-growing mycobacteria plasmid - secretion system - conjugation

Plasmids are genetic elements that make up bacteria mobilome involved in horizontal gene transfer (HGT) events. Conjugative plasmids are characterised by the presence of the mobility (MOB) genes region, defined by the origin of replication (oriT) and a relaxase gene, and the type IV secretion system (T4SS)-like genes (Smillie et al. 2010).

The Mycobacterium genus encompasses more than a hundred species including fast- and slow-growing organisms, and the latter contains the majority of opportunistic and human pathogens. However, only few plasmids have been described in mycobacteria, and conjugative elements have been rarely reported (Ummels et al. 2014, Uchiya et al. 2015). In fact, conjugation in mycobacteria is just being elucidated (Gray et al. 2016). Interestingly, some mycobacteria plasmids carry genes resembling the type VII secretion system (T7SS) or ESX-P, which is the unique secretion system recognised in Mycobacterium genus. In Mycobacterium tuberculosis five ESX types (ESX-1-5) were identified based on the ESX core components (EccB, EccC, EccD, and MycP) and on particular variations in the gene content and organisation (Costa et al. 2015, Simeone et al. 2015). The ESX types evolved by gene duplication, and plasmids have been proposed to be a key factor in their radiation into this genus (Dumas et al. 2016, Newton-Foot et al. 2016).

In this study, we determined the complete sequence of pCBMA213_2 plasmid (accession no. KY349138) carried by an environmental fast-growing Mycobacteria strain. The Mycobacterium sp. CBMA213 was isolated from the Atlantic Forest soil and characterised by

doi: 10.1590/0074-02760160546

Financial support: CAPES, PNPD-CAPES (fellowships), CNPq, IOCFIOCRUZ (grants).

+ Corresponding author: sergio.morgado@ioc.fiocruz.br

Received 20 December 2016

Accepted 28 January 2017 multilocus sequence analysis (MLSA) as belonging to a new species of Mycobacterium (data not shown). The pCBMA213_2 sequence was obtained in the context of the CBMA213 whole genome sequencing, using Nextera paired-end library on Illumina Hiseq 2500. Reads were filtered and correted using NGSQCToolkit v.2.3.3 (Patel \& Jain 2012) and Quake v.0.3 (Kelley et al. 2010). De novo assembly was done using SPAdes v.3.9 (Bankevich et al. 2012). A total of 57 contigs were obtained, and among them, a $160 \mathrm{~kb}$-contig was putatively characterised as a plasmid by the presence of mycobacteria plasmid gene markers, such as relaxase and repA (Ummels et al. 2014, Uchiya et al. 2015). Moreover, its circular topology defined by paired-end information characterised this contig as a replicon. This plasmid was mapped and reassembled with a mean coverage of $144 x$. A final step of correction was performed using Pilon v.1.20 (Walker et al. 2014). A Maximum Likelihood phylogeny was constructed based on concatenated EccA-E and MycP amino acid sequences (4,700 aa) using Seaview v.4.6.1 (Gouy et al. 2010).

The pCBMA213_2 is a circular plasmid of 160,489 $\mathrm{bp}$, with a guanine-cytosine content (GC content) of $65.9 \%$, characteristic of Mycobacterium genus and similar to GC content $(65.4 \%)$ of CBMA213 genome. The annotation using Prokka v.1.11 (Seemann 2014) identified 161 coding DNA sequence (CDS), and BLASTn analyses revealed that pCBMA213_2 was unique relative to other mycobacterial plasmids considering the overall gene content. However, genes sharing identity with elements related with plasmid conjugation (virD4, virB $4, t c p C)$ and mobilisation (relaxase), as well as a set of genes resembling an entire T7SS, were identified among these 161 CDS (Fig. 1). The pCBMA213_2 T7SS genes share the same synteny and $68 \%$ identity with the Mycobacterium $s p$. KMS pMKMS01. Indeed, a phylogenetic reconstruction based on EccA-E and MycP proteins, recovered from mycobacteria NCBI database, reveled that pCBMA213_2 belongs to a cluster (ESX-P2), with three other mycobacteria plasmids, and this cluster is related to M. tuberculosis chromosomal ESX-2 system (Fig. 2). 


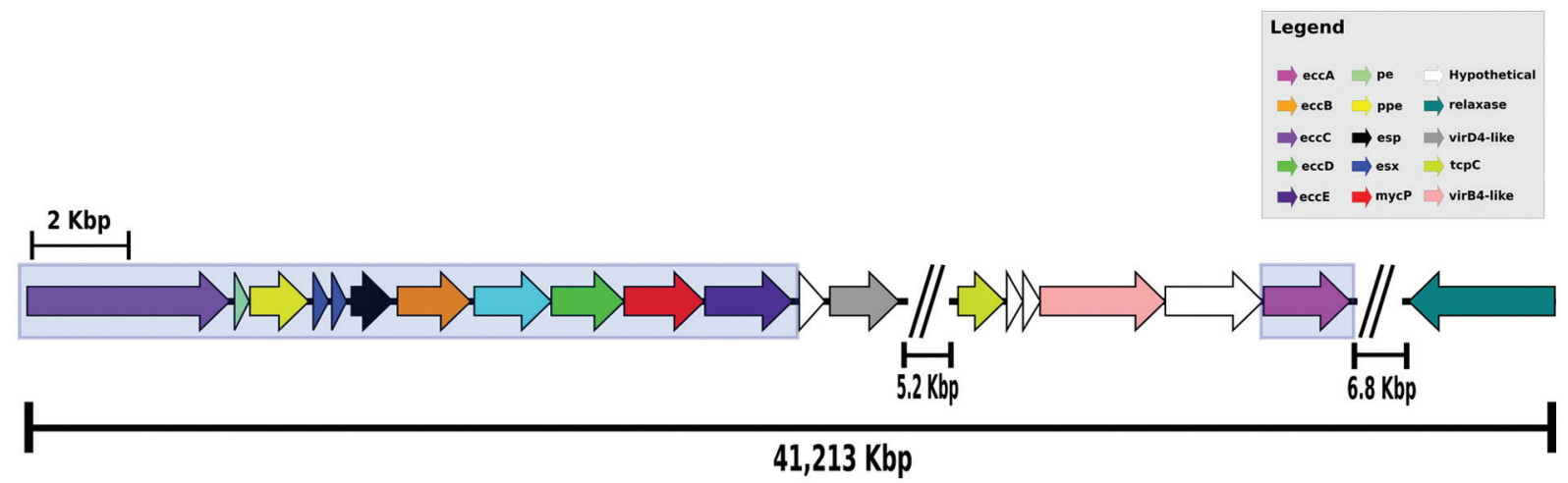

Fig. 1: gene organisation of T7SS, T4S-like and relaxase loci, separated by segments with hypothetical genes, in pCBMA213_2. T7SS genes are depicted by box. This segment contains $41,213 \mathrm{~kb}$.

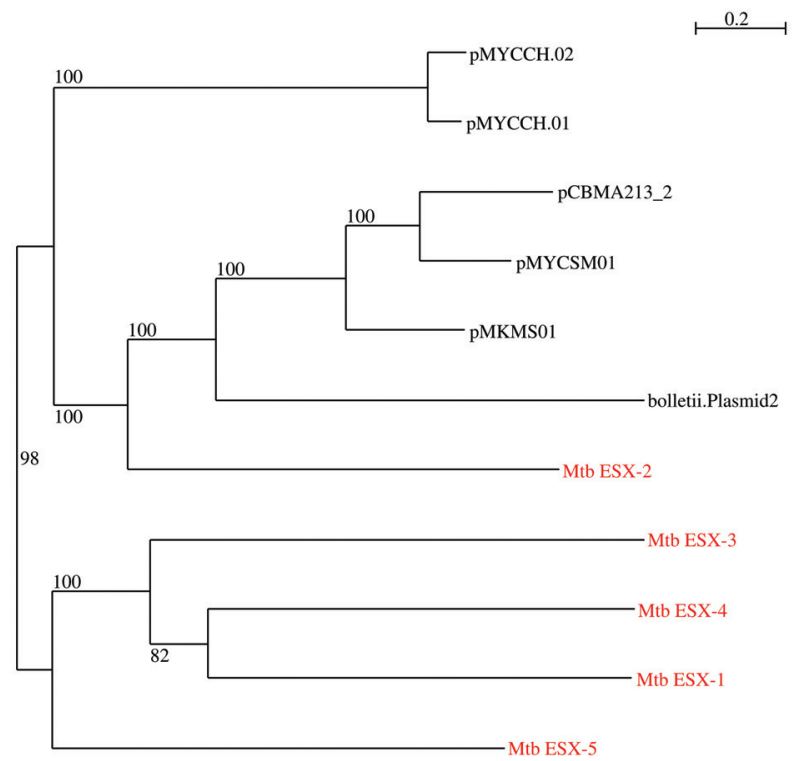

Fig. 2: maximum likelihood phylogenetic tree of T7SS loci from mycobacteria plasmids and M. tuberculosis (Mtb), based on concatenated EccA-E and MycP amino acid sequences (100 bootstrap replications). Chromosomal ESX 1-5 is colored in red.

In relation to $\mathrm{T} 4 \mathrm{~S}$ conjugation system, homologues of traI relaxase ( $\left.p C B M A 213 \_2 \_00112\right)$, virB4 ( $p C B$ MA213_2_00101), virD4 ( $\left.p \bar{C} B \bar{M} A 213 \_2 \_00091\right)$ and tcp C ( $\bar{p} C \bar{B} M A 213$ 2 00098$)$ genes are encoded in pCBMA213_2, but some other T4SS components were not identified. The genes from both secretion systems are distributed in a $41,213 \mathrm{~kb}$ segment. Interestingly, Ummels et al. (2014) characterised a novel functional conjugative plasmid in a M. marinum that combines elements from both type IV and type VII secretion systems enabling its transfer only between slow-growing mycobacteria. The pCBMA213_2, harbored by a fast-growing mycobacteria, contains this same set of elements, indicating that this plasmid belongs to this new class of conjugative plasmid.

Noteworthy, this plasmid carries the whiB6 ( $p C B$ MA213_2_00051) that has been recently demonstrated to be involved in the regulation of ESAT- 6 production and secretion. ESAT- 6 is the virulence factor secreted by ESX-1 secretion system in M. tuberculosis (Solans et al. 2014, Chen et al. 2016).

Considering the mycobacteria plasmids available at GenBank, pCBMA213_2 presented the highest identity and coverage with pMYCSM01 from a fast growing $M$. smegmatis strain isolated from a human soft tissue lesion (Fig. 2). They share high identity within T7SS loci subtilisin gene and three hypothetical protein genes.

Therefore, pCBMA213 2 can be further explored as a model for conjugation studies and/or a tool to introduce DNA in fast-growing mycobacteria.

\section{ACKNOWLEDGEMENTS}

To the Oswaldo Cruz Foundation (FIOCRUZ) high-throughput sequencing platform.

\section{AUTHORS' CONTRIBUTION}

SMM - Performed the in silico and in vitro analyses, discussed the results and wrote the paper; MAM - contributed with the in silico experimental design, discussed the in silico results; FSF performed the microbiological assays; ELF - contributed with the discussion of the results; ACPV - conceived and supervised all steps of the study, discussed the results and wrote the paper.

\section{REFERENCES}

Bankevich A, Nurk S, Antipov D, Gurevich AA, Dvorkin M, Kulikov AS, et al. SPAdes: a new genome assembly algorithm and its applications to single-cell sequencing. J Comput Biol. 2012; 19(5): 455-77.

Chen Z, Hu Y, Cumming BM, Lu P, Feng L, Deng J, et al. Mycobacterial WhiB6 differentially regulates ESX-1 and the Dos regulon to modulate granuloma formation and virulence in zebrafish. Cell Rep. 2016; 16(9): 2512-24.

Costa TR, Felisberto-Rodrigues C, Meir A, Prevost MS, Redzej A, Trokter M, et al. Secretion systems in gram-negative bacteria: structural and mechanistic insights. Nat Rev Microbiol. 2015; 13(6): 343-59.

Dumas E, Christina EB, Vandenbogaert M, de la Vega RCR, Thiberge JM, Caro V, et al. Mycobacterial pan-genome analysis suggests important role of plasmids in the radiation of type VII secretion systems. Genome Biol Evol. 2016; 8(2): 387-402.

Gouy M, Guindon S, Gascuel O. SeaView version 4: a multiplatform graphical user interface for sequence alignment and phylogenetic tree building. Mol Biol Evol. 2010; 27(2): 221-4. 
Gray TA, Clark RR, Boucher N, Lapierre P, Smith C, Derbyshire KM. Intercellular communication and conjugation are mediated by ESX secretion systems in mycobacteria. Science. 2016; 354(6310): 347-50.

Kelley DR, Schatz MC, Salzberg SL. Quake: quality-aware detection and correction of sequencing errors. Genome Biol. 2010; 11(11): R116.

Newton-Foot M, Warren RM, Sampson SL, van Helden PD, van Pittius NCG. The plasmid-mediated evolution of the mycobacterial ESX (Type VII) secretion systems. BMC Evol Biol. 2016; 16: 62.

Patel RK, Jain M. NGS QC toolkit: A toolkit for quality control of next generation sequencing data. PLoS ONE. 2012; 7(2): e30619.

Seemann T. Prokka: rapid prokaryotic genome annotation. Bioinformatics. 2014; 30(14): 2068-9.

Simeone R, Bottai D, Frigui W, Majlessi L, Brosch R. ESX/type VII secretion systems of mycobacteria: insights into evolution, pathogenicity and protection. Tuberculosis (Edinb). 2015; 95(Suppl. 1): S150-4.
Smillie C, Garcillán-Barcia MP, Francia MV, Rocha EP, de la Cruz F. Mobility of plasmids. Microbiol Mol Biol Rev. 2010; 74(3): 434-52.

Solans L, Aguiló N, Samper S, Pawlik A, Frigui W, Martín C, et al. A specific polymorphism in Mycobacterium tuberculosis $\mathrm{H} 37 \mathrm{Rv}$ causes differential ESAT-6 expression and identifies WhiB6 as a novel ESX-1 component. Infect Immun. 2014; 82(8): 3446-56.

Uchiya K, Takahashi H, Nakagawa T, Yagi T, Moriyama M, Inagaki T, et al. Characterization of a novel plasmid, pMAH135, from Mycobacterium avium subsp. hominissuis. PLoS ONE. 2015; 10(2): e0117797.

Ummels R, Abdallah AM, Kuiper V, Aâjoud A, Sparrius M, Naeem $\mathrm{R}$, et al. Identification of a novel conjugative plasmid in mycobacteria that requires both type IV and type VII secretion. mBio. 2014; 5(5): e01744-14.

Walker BJ, Abeel T, Shea T, Priest M, Abouelliel A, Sakthikumar S, et al. Pilon: an integrated tool for comprehensive microbial variant detection and genome assembly improvement. PLoS ONE. 2014; 9(11): e112963. 